\title{
REVIEW
}

\section{Dementia-related agitation: a review of non-pharmacological interventions and analysis of risks and benefits of pharmacotherapy}

\author{
EO ljaopo
}

Unsurprisingly, the subject of dementia has been a rising matter of public health concerns as people now live longer. World Alzheimer Report 2015, estimate that about 46.8 million people worldwide have dementia. These numbers are projected to almost double every 20 years, reaching 74.7 million in 2030 and 131.5 million in 2050. The modality for treating agitation and other behavioral symptoms in dementia patients has been a challenge. Many years on, there has been no FDA-approved pharmacotherapy in treating dementia-related agitation. This review discusses the current knowledge of non-pharmacological interventions, and analyzes the risks and benefits of pharmacotherapy in the management of dementia-related agitation, as well as providing an anecdotal of the author's clinical experience. This article aims to provide opportunity for increase awareness for clinicians, particularly those with no specialty training in geriatrics medicine but see dementia patients with agitation and other behavioral symptoms from time to time. Likewise, it hopefully will benefit the readers of medical journals to update their existing knowledge on matters relating to the management of dementia-related agitation.

Translational Psychiatry (2017) 7, e1250; doi:10.1038/tp.2017.199; published online 31 October 2017

\section{INTRODUCTION}

Because of increased longevity, the number of people being diagnosed with dementia is on the rise. In the year 2015 alone, an estimated 9.9 million new cases of dementia was expected to be diagnosed worldwide, equivalent to one new case of dementia in every 3.2 s. ${ }^{1}$ World Alzheimer Report in 2015 estimates about 46.8 million people worldwide have dementia. These numbers are projected to almost double every 20 years, reaching 74.7 million in 2030 and 131.5 million in 2050. In the United States of America, the Alzheimer's Association claims over 5 million of Americans have dementia, and by the year 2050, it is estimated that up to 16 million Americans will have dementia. ${ }^{2}$

The 2015 global societal economic cost of dementia was estimated at US\$818bn, and this is expected to rise to a trillion dollars by the year 2018. ${ }^{1}$ In the United States, in 2010, the yearly monetary cost per person with dementia varied from $\$ 41689$ to $\$ 56$ $290 .^{3}$ The American Alzheimer's Association claim the monetary costs alone attributable to dementia were $\$ 172$ billion. ${ }^{4}$

Unfortunately, during the course of their disease, over $90 \%$ of dementia patients will suffer from psychiatric or behavioral problems commonly referred to as neuropsychiatric symptoms. ${ }^{5,6}$ Neuropsychiatric symptoms in dementia patients include agitation, delusions, hallucinations, dysphoria, anxiety, aggression, euphoria, disinhibition, irritability/lability, apathy and aberrant motor activity that include wandering. ${ }^{7,8}$ Agitation, being one of the most commonly observed neuropsychiatric symptoms, is reported to be found in up to $70 \%$ of dementia patients. ${ }^{9-11} \mathrm{~A}$ longitudinal observational study in Dutch nursing homes, where 372 residents with dementia were followed up for 3.5 years (20072011 ) to assess the prevalence and course of symptoms exhibited during dementia illness from the time of admission into care facility to the time of death, found agitation to be the most common prevalent symptom, accounting for up to $71 \% .^{12}$ Agitation can be described as restless behavior, or improper physical and verbal actions that may cause trouble for family members, caregivers and other service users. Quite commonly, it is associated with caregiver burden that results in the caregivers getting burnout. ${ }^{13}$ Likewise, agitation in one resident may trigger agitation in the other residents. ${ }^{14}$

Recommended management for dementia-related agitation and other behavioral symptoms involve non-pharmacological interventions and pharmacotherapy. The American (including the American Associations for Geriatric Psychiatry), British and Canadian Geriatrics Societies recommend non-pharmacological interventions as the first-choice approach in managing dementiarelated agitation. However, in emergency situations when nonpharmacological approach is not feasible due to an imminent danger to patient's safety, drug therapy is recommended as a firstline treatment intervention. However, many years on, there is still no Food and Drug Administration (FDA)-pharmacotherapy in treating dementia-related agitation. This review discusses the current knowledge of non-pharmacological interventions and analyses the risks and benefits associated with pharmacotherapy in the management of dementia-related agitation, as well as providing an anecdotal of the author's clinical experience.

\section{METHODS}

A computer search of databases including PubMed, MEDLINE, CINAHL, Psyclnfo and EMBASE were carried out to identify studies 
in peer-reviewed journals published within the last dozen years, from January, 2005 to March, 2017. Search terms used were: treatment for dementia agitation, drugs treatment/pharmacotherapy and non-pharmacological treatment/interventions, management of neuropsychiatric behavior in dementia (because some studies included agitation as part of the neuropsychiatric behavior). Systematic reviews, meta-analyses and randomized trials (of pharmacologic and non-pharmacologic treatments), were included for review. Likewise, practical guidelines for dementiarelated agitation were review. A manual search of additional references was also done among the references found in the databases. Clinical trials.gov was also reviewed for completed and ongoing trials related to treatment of agitation in dementia. Only studies conducted in English language were preferentially reviewed. Data synthesis and recommendations from this review came from available evidence obtained from the studies reviewed and the anecdotal clinical experience of the author.

\section{NON-PHARMACOLOGICAL INTERVENTIONS}

The growing concerns over the efficacy and side effects of pharmacotherapy have resulted in the emphasis of nonpharmacological interventions as the first line treatment approach.

NICE UK in agreement with the American and the Canadian Association for Geriatric Psychiatry, as well as the European Association of Geriatric Psychiatry (EAGP) recommends the initial treatment approach for people who have agitation in all types and severities of dementia to be the non-pharmacological interventions. $^{33}$

This approach includes simple and complex interventions which are most often combined and provided to patients in a personcentered care approach. Examples of these interventions include multisensory stimulation, aromatherapy, music therapy, cognitive behavioral therapy, animal-assisted therapy, electroconvulsive therapy (ECT) and physical exercises. These non-pharmacological interventions are increasingly being recognized globally as the crucial parts of the comprehensive dementia care due to its fewer risks compare to the drugs treatment. Unenthusiastically however, available evidence still shows that effective non-pharmacological interventions have not been widely embraced by the real-world clinical practice and standard of care. ${ }^{10,18,34-36}$ Kales et al. recommend a non-pharmacologic strategy called DICE approach to be used as a basis for integrating non-pharmacologic and pharmacologic interventions in the real-world clinical settings. ${ }^{35}$ The DICE approach which comprises Describe, Investigate, Create and Evaluate, is essentially patient/caregiver-centered approach that describes sequential steps for thorough assessments to help manage neuropsychiatric symptoms of dementia. Kales et al. claim DICE model will provide clinicians with well-planned efficient treatment approach when integrated into clinical practice. Another model called Targeted Interdisciplinary Model for Evaluation and treatment of neuropsychiatric symptoms (TIME) was described by Lichtwarck et al. as a multicomponent intervention program frequently employed by physicians and nursing home staff (in Norway) for assessing and treating behavioral and psychological symptoms of dementia or other complex disorders. ${ }^{37}$ The TIME model consists of three interconnected phases of registration and assessment phase; a guided reflection phase; and an action and evaluation phase. These phases were extracted from the concepts of cognitive behavioral therapy and are to be tailored to individual patient. In 2016, Lichtwarck et al., published a study protocol on the proposed 3month TIME trial to take place in 30 nursing homes (in Norway) involving 168 residents with high degree of dementia-related agitation, so as to evaluate the effectiveness-implementation of TIME model and its implementation process by staffs and at organization levels. The authors believe the result of TIME intervention model could become an evidence-based model that will improve assessment and treatment of agitation and other neuropsychiatry symptoms in dementia patients.

Within the last decade alone, numerous studies (Abraha et al.; ${ }^{19}$ Cohen-Mansfield et al.;8 Rapp et al.;9 ${ }^{39}$ Blythe et al.; ${ }^{40}$ Cooke et al.; ${ }^{41}$ Jutkowitz et al. ${ }^{22}$ Lin et al.; ${ }^{42}$ van der Ploeg et al.; ${ }^{43}$ Van Vracem et $a l^{44}$ ) have been published describing the effectiveness of the non-pharmacologic interventions in the prevention and treatment of dementia-related agitation (Tables 1 and 2).

Hawranik et al. ${ }^{15}$ and Woods et al. ${ }^{16}$ describe the potential usefulness of therapeutic touch as playing significant positive role in managing dementia-related agitation and other behavioral symptoms. The combination of therapeutic touch using acupressure and psychosocial interventions using Montessori activities also recorded positive findings toward decreasing agitation in residents with dementia. ${ }^{45}$ Listening to familiar music is shown to demonstrate significant effectiveness in reducing agitation and other related behavioral and psychiatric symptoms in patients with moderate to severe dementia. ${ }^{46,47}$ Also, proven to be of significant clinical benefit in reducing agitation are the tailored activities program channeled to the capability of individual dementia patients. ${ }^{48}$ Gitlin et al. ${ }^{48}$ add that the tailored activities training provided to families/caregivers also result in beneficial effects which reduce caregiver burden and improved skills among other benefits.

Conventionally, it is long believed that lavender has some of therapeutic and curative properties. Neurophysiological and animal studies according to Koulivand et al. ${ }^{49}$ report that lavender oil may have some effectiveness in the treating several neurological disorders due to its anxiolytic, sedative and neuroprotective properties. During that interval, O'Connor et $a l^{17}{ }^{17}$ undertook a randomized single-blind cross-over trial of dermally-applied, physiologically active, high purity $30 \%$ lavender oil versus an inactive control oil on 64 nursing home residents with frequent physically agitated behaviors. They found that despite its sedative and anxiolytic properties, lavender oil showed no significant advantage over control oil and demonstrated no evidence in reducing the agitation behavior in dementia patients.

Further, in 2014, Livingston et al. ${ }^{18}$ conducted systematic review of 160 studies that investigated non-pharmacological interventions for agitation in dementia people over 50 years of age in care facility settings. They reported that engaging agitated dementia patients on various activities including music therapy and sensory interventions (massage, therapeutic touch and multisensory stimulation) may help to reduce mild-to-moderate agitation in the immediate term, but, lacked significant long term benefits, and no beneficial effects on severe agitation symptoms. However, caregivers trained and supervised in communication or personcentered skills demonstrated immediate improvement on severe agitation with effects lasting up to 6 months. In contrary, light therapy (of 30-60 min daily bright-light exposure) and aromatherapy interventions reported no evidence of benefit in reducing agitation.

Meanwhile, as agitation in dementia patients may be due to other causes, focusing to alleviate pain and sleep disorders problems, and discontinuation of inappropriate medications may markedly help the management of behavioral symptoms related to dementia. ${ }^{50}$ Likewise, dealing with hypothesized unmet needs of dementia patients play remarkable role in managing agitationrelated symptoms in dementia. ${ }^{38}$ The latter point was further buttressed by Jakobson et al. ${ }^{10}$ who demonstrated that patient specific intervention programs that aim to address unmet needs have proven evidence in reducing agitation in dementia patients.

Abraha et al. ${ }^{19}$ present an overview of nonpharmacological interventions for behavioral and psychological symptoms in dementia through systematic overview of 38 systematic reviews and 142 primary studies comprising different categories of nonpharmacological interventions. ${ }^{19}$ They studied various sensory 


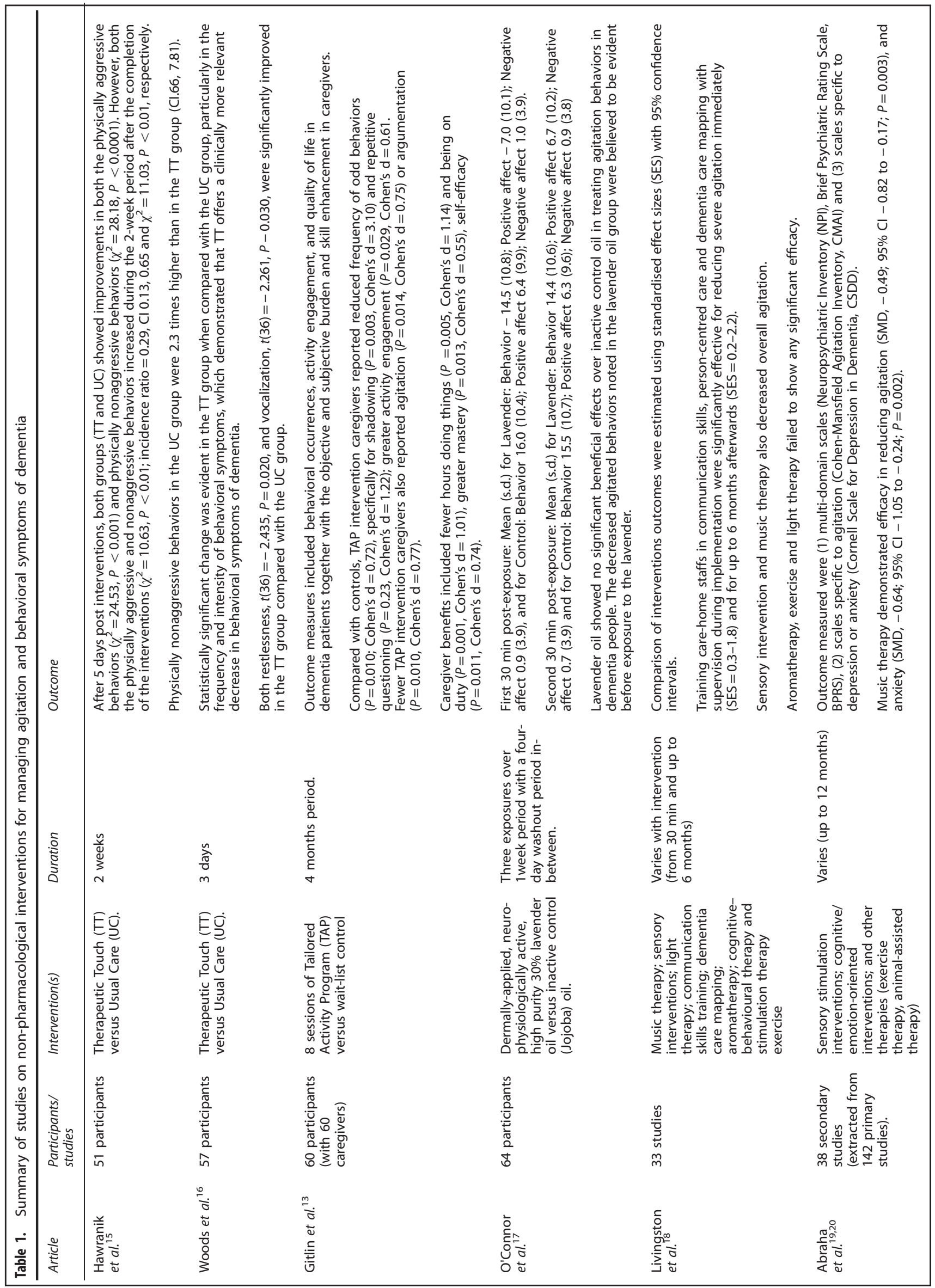




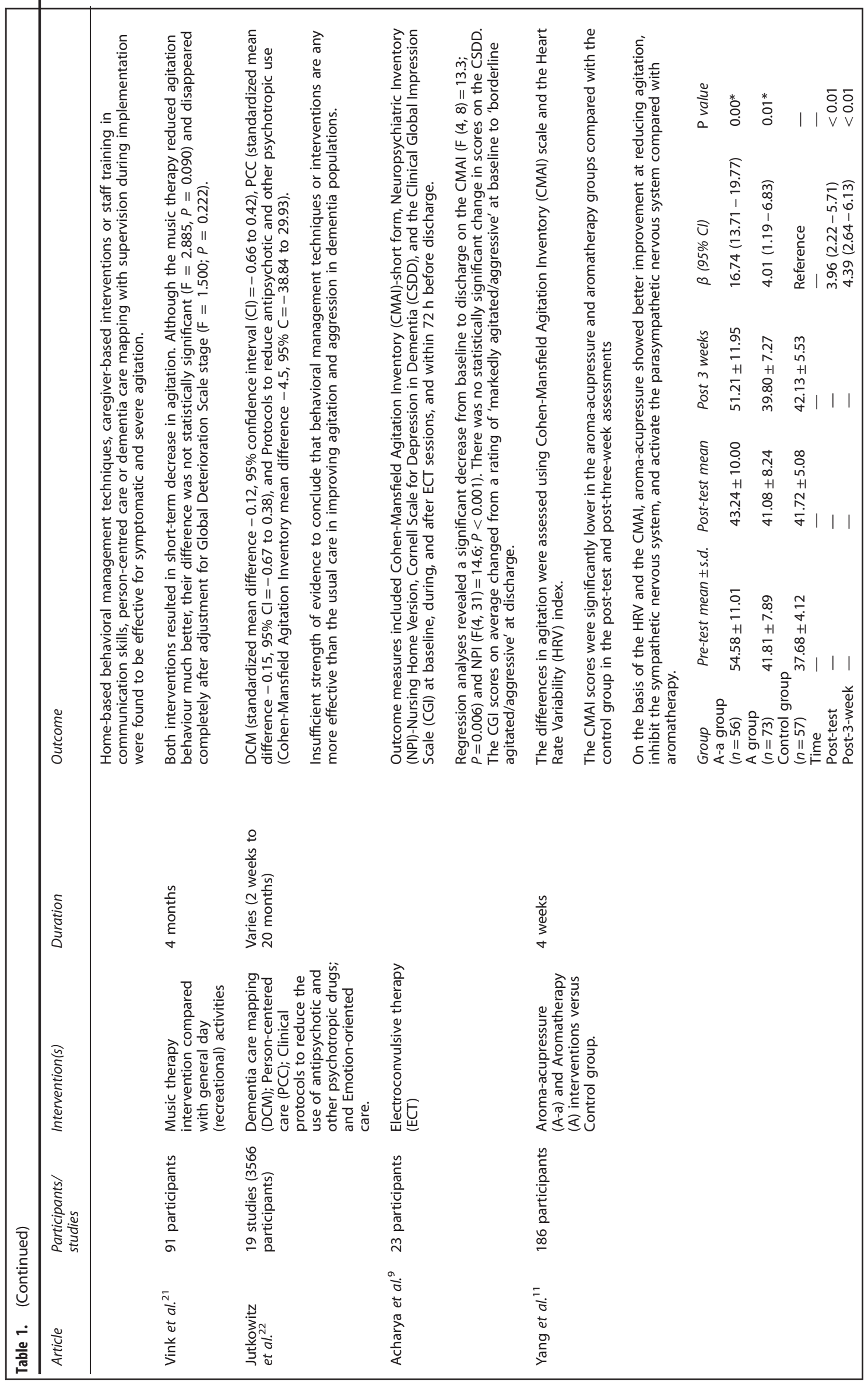




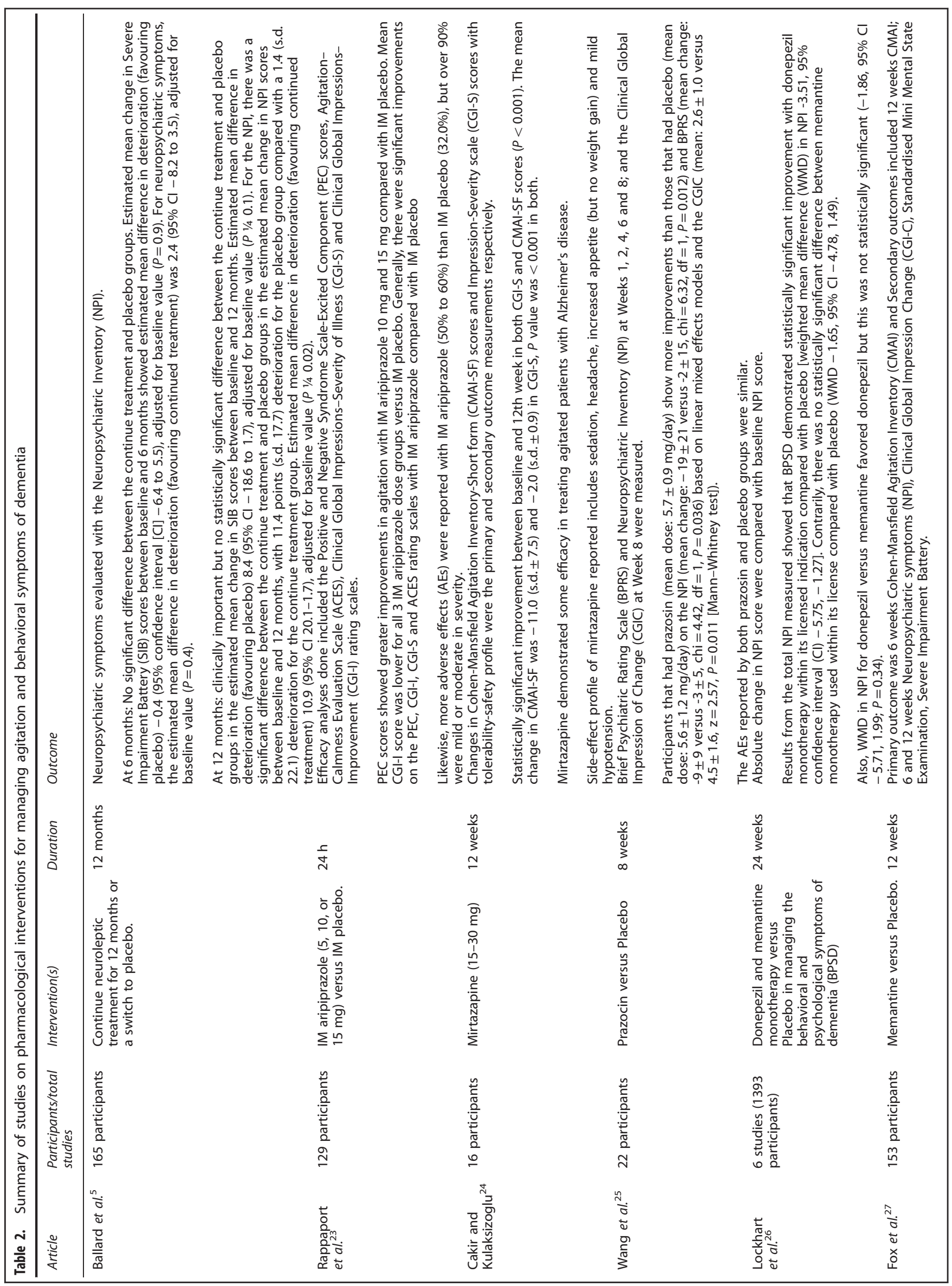




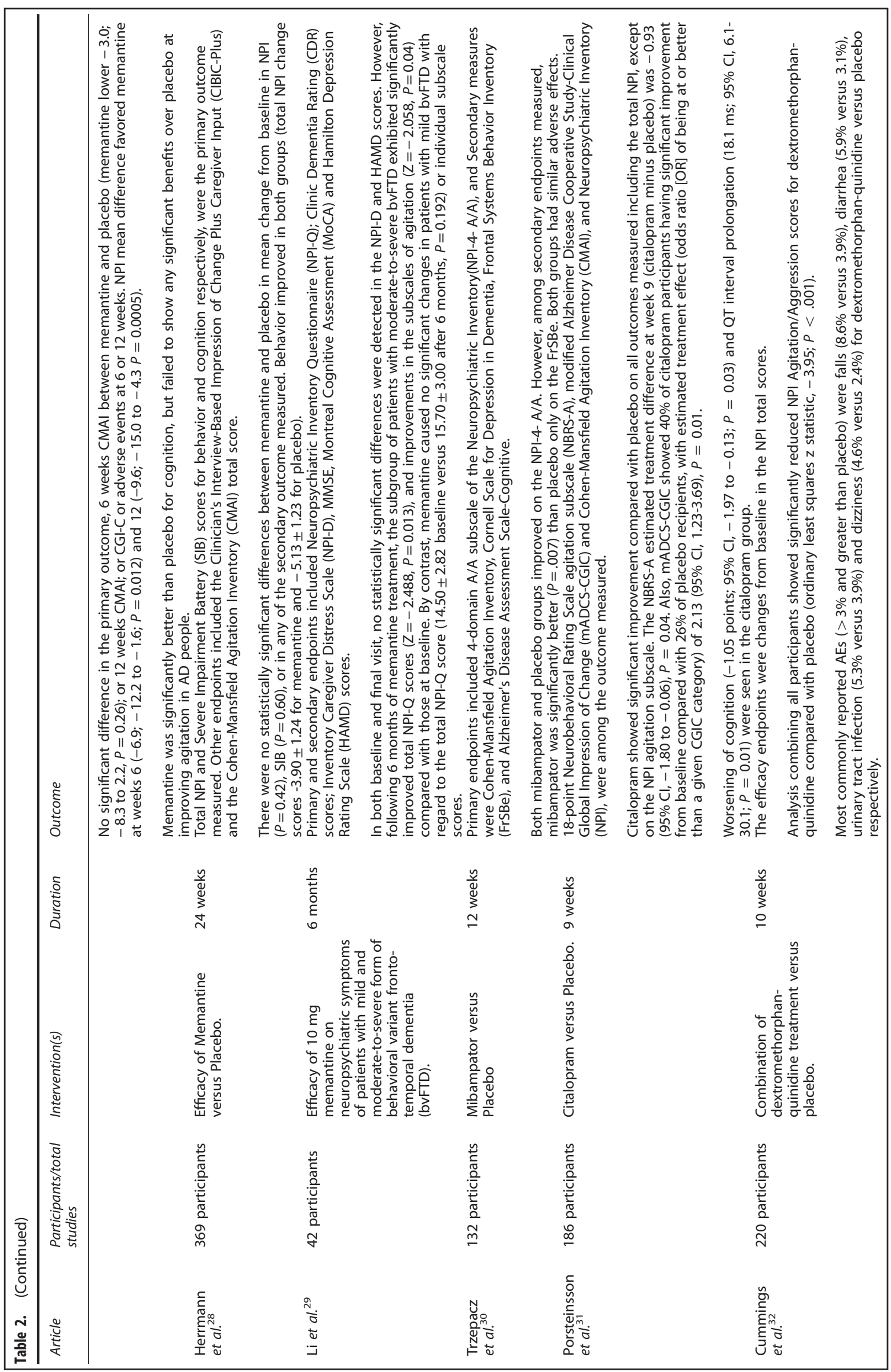


stimulation interventions (acupuncture, aromatherapy, massage therapy, light therapy, sensory garden intervention, cognitive stimulation, music/singing and dance therapy, snoezelen and transcutaneous electrical nerve stimulation (TENS) therapy); with cognitive/emotion-oriented interventions; behavioral management technique; and other interventions including exercise therapy, pet-therapy and special care unit. Abraha et al. ${ }^{19}$ found that music therapy is the only effective nonpharmacological sensory intervention that reduces agitation in dementia patients. This finding is corroborated by other previous studies (Ridder et al.; ${ }^{14}$ Sung and Chang; ${ }^{47}$ Ray and Mittelman ${ }^{51}$ ), which demonstrated significant beneficial effects of music therapy in reducing symptoms of agitation in dementia patients. Meanwhile, in another randomized trial by Vink et al. ${ }^{21}$ comparing the effects of music therapy with general day activities on agitation in 77 dementia persons resident in nursing home, claimed that both music therapy and recreational activities only resulted in shortterm decrease in agitation, and that music therapy did not show any additional beneficial effects over general activities.

Further analysis by Abraha et al. ${ }^{19}$ claims that some behavioral management techniques directed at enhancing staff communication skills, formal caregiver training and dementia mapping were effective at reducing agitation. But, interventions such as exercise and pet therapy failed to show any beneficial effects on the behavioral and psychological symptoms of dementia patients. Meanwhile, quite oppositely, a systematic review and metaanalysis of nineteen studies by Jutkowitz et al. $^{22}$ to evaluate the efficacy of selected nonpharmacological care-delivery interventions including staff training, care-delivery models and environment changes in reducing agitation and aggression in care home dementia residents found insufficient evidence to prove that the behavioral management techniques are any more effective than the usual care in improving agitation and aggression in dementia patients.

When assessing the safety of ECT, Acharya et al. ${ }^{9}$ analyzed 23 subjects who had 12 ECT courses to treat their symptoms of agitation and aggression. They report that the use of antipsychotic medications for agitation and aggression symptoms was significantly reduced during the period of the ECT treatment. Acharya et al. ${ }^{9}$ suggest that ECT may be considered a suitable, safe and effective treatment for some patients with dementia whose behavioral disturbances are unresponsive to the standard nonpharmacological interventions and pharmacotherapy, or are not able to tolerate the latter.

In another experimental study, Yang et al. ${ }^{11}$ assess the efficacy of aroma-acupressure and aromatherapy in the treatment of dementia-related agitation. Although agitation improved in both groups, aroma-acupressure showed a better effect than aromatherapy in reducing agitation in dementia patients. They recommend future studies are necessary to further explore the benefits of aroma-acupressure and aromatherapy in treating agitation in dementia patients.

Several further studies have discussed various positive findings that promote the safety and efficacy of non-pharmacological approaches at improving dementia-related agitations in care facilities. ${ }^{8,36,52}$ Although, a recent review by Abraha et $a .^{20}$ that analyzes three studies of differing design, involving 144 dementia persons, assessing whether simulated presence therapy intervention using video or audiotape recordings of family members played to dementia person, helps in reducing agitation and distressed symptoms. They concluded there to be inadequate evidence available to believe simulated presence therapy intervention has significant efficacy in treating behavioral and psychiatric symptoms of dementia patients.

Despite the general recommendation of non-pharmacological approach as the first-line choice in treating dementia-related agitation and the available evidences supporting some of them, a survey study conducted by Cohen-Mansfield et $a l^{53}$ in Israeli nursing homes, to examine physicians' actual practice in treating dementia-related agitation and to check their familiarity with use of non-pharmacological interventions showed that $92.5 \%$ of physicians prescribed psychotropic medications for treating agitation. Quite astonishingly, the 67 physicians studied show low-familiarity levels with the use of non-pharmacological interventions. More so, as can be predicted, physicians with non-specialty in geriatrics were noted to have higher levels of unfamiliarity with non-pharmacological interventions.

With so much being said, the non-pharmacologic interventions are not without challenges. They are sometimes more difficult to carry out and could take longer time to be effective. They are also best tailored to individual clinical needs. Little wonder, their uptake as the preferred treatments for dementia-related agitation and neuropsychiatric symptoms remains limited in the real-world clinical settings. More so, even as most evidence of nonpharmacologic interventions available from literatures show good efficacy in the early and mid-stages of dementia, whether these interventions are effective in the later stages of dementia remains unclear. ${ }^{54}$

Finally, it is important to note that agitation may occur as an end-of-life symptom in people with advanced dementia, Eapcnet. $\mathrm{eu}^{55}$ discusses the palliative care approach to managing behavioral and psychological symptoms of dementia (BPSD) which may include agitation behavior that challenges caregivers, or be distressing for the patient. In these situations, they recommend getting the opinions of geriatricians or dementia care/palliative care specialists. van der Steen et al. ${ }^{56}$ further elaborated on the European Association for Palliative Care (EAPC) recommendations and provide a framework guidance for clinical practice, research and policy. To further buttress the need for palliative care approach in managing agitation symptoms in advanced dementia, Hendriks et $a l^{57}$, reviewed the qualities of life of 330 nursing home residents with dementia who were in their last week of life and found that $35 \%$ of them had agitation symptoms.

\section{RISKS AND BENEFITS OF PHARMACOLOGICAL INTERVENTIONS}

Quite often, it is not uncommon that physicians use pharmacotherapy approach for treating dementia-related agitation and other neuropsychiatric symptoms (NPS). Several drugs including neuroleptics, anti-depressants, sedatives/hypnotics and anxiolytics among others are frequently used. While there is still no FDAapproved drug treatment for dementia-related agitation, they have issued several black box warnings regarding increased risk of stroke and mortality associated with the use of some drugs (particularly antipsychotics) in the elderly patients with dementia. ${ }^{31,58}$

Ballard et al. ${ }^{5}$ conducted a randomized trial across five areas in Great Britain, to examine dementia patients who were started on long-term (at least 3 months) neuroleptic agents for neuropsychiatric symptoms control. They assess whether neuroleptics affect cognitive function and other outcomes, and determine whether discontinuing neuroleptics was related to an exacerbation of neuropsychiatric symptoms. Ballard et al. ${ }^{5}$ report that withdrawal of neuroleptics in most patients with Alzheimer's disease led to measurable improvement of cognitive and functional status, and no significant harmful effect. A year later, in 2009, Rappaport et al. ${ }^{23}$ did a randomized, double-blind, placebo-controlled tolerability study of intramuscular aripiprazole on 129 acutely agitated patients with Alzheimer's, vascular, or mixed dementia across 16 centers in the USA. Moderate-to severe acute exacerbations of agitated behavior was defined using Positive and Negative Syndrome Scale-Excited Component (PEC) scores, and they performed efficacy analyses for the PEC, Agitation-Calmness Evaluation Scale (ACES), Clinical Global Impressions-Severity of Illness (CGI-S) and Clinical Global 
Impressions-Improvement (CGI-I) rating scales. The authors concluded that a total of $10 \mathrm{mg}$ or $15 \mathrm{mg}$ of intramuscular aripiprazole given in divided doses showed overall good tolerability and remarkable improvements in agitation associated with Alzheimer's, vascular, or mixed dementia. Similarly, another study by Seitz et al. ${ }^{59}$ on several atypical antipsychotics (risperidone, olanzapine and aripiprazole) showed demonstrable good evidence in managing neuropsychiatric symptoms of dementia patients. But, Seitz et al. ${ }^{59}$ recommend that safe and effective non-pharmacological interventions approach should be considered ahead of antipsychotics and other pharmacological treatments due to distressing adverse effects. Schneider et al. ${ }^{60}$ demonstrated that atypical antipsychotics are associated with an increased risk of death, with an odds ratio of 1.54. Likewise, observational study by Gill et al. ${ }^{61}$ found an increased risk of mortality with the use of the antipsychotics. There is also reported evidence of increased risk of major cerebrovascular events associated with antipsychotics use. ${ }^{62}$ Other common adverse effects which should also be monitored during treatment with atypical antipsychotics include increased falls and fall-related injuries such as hip fractures. ${ }^{63}$ Most often, these risks occur soon after initiating antipsychotics treatment, although chronic therapy is also argued to be associated with increased risks. ${ }^{5,54}$

In a 12-week prospective study involving sixteen Alzheimer's disease (AD) patients with significant agitation by Cakir and Kulaksizoglu, ${ }^{24}$ it was believed that mirtazapine has some effectiveness in treating agitation related to AD. Mild side-effects such as sedation, headache, increased appetite and mild hypotension relating to mirtazapine were reported in the study. Comparably, Wang et al. ${ }^{25}$ study of 22 nursing home and community dwelling patients with probable Alzheimer's disease who had agitation and aggression randomized to an 8-week single-site study of placebo versus prazosin concluded that prazosin, compared with placebo, demonstrates remarkable improvement at reducing agitation and aggression in AD patients. They observed that prazosin was well tolerated by the participants and very rarely associated with sedation which is one of the worrying side effects of neuroleptics.

Donepezil and memantine are frequently used in mild-tomoderate and moderate-to-severe symptoms of AD respectively. Their roles as monotherapies in managing the BPSD within their licensed indications were compared in a systemic review and meta-analytic study by Lockhart et al. ${ }^{26}$ analyzed six randomized controlled monotherapy trials (four donepezil and two memantine) and findings showed that donepezil monotherapy within its licensed indication is found to be significantly more efficacious than placebo for alleviating BPSD. On the other hand, memantine monotherapy within its licensed indication failed to show any statistically significant advantage over placebo in managing BPSD. Fox et al. ${ }^{27}$ findings also buttressed the claim that memantine has no significant advantage over placebo in improving agitation in people with moderate-to-severe dementia. Exploring efficacy of memantine further, Herrmann et al., ${ }^{28}$ organized a randomized, double-blind placebo-controlled trial on 369 patients with moderate-to-severe AD over 24 weeks period to assess the efficacy of memantine over placebo in reducing agitation and aggression-related symptoms. Several standardized tests scores (see Appendix) employed to measure the outcome showed no statistically significant benefit of memantine over placebo in reducing the symptoms of agitation and aggression in moderateto-severe AD. Quite contrarily, Li et al., ${ }^{29}$ claim memantine treatment has beneficial effects on neuropsychiatric symptoms of patients with moderate-to-severe form of behavioral variant frontotemporal dementia (bvFTD) after conducting a six-month, open-label, self-controlled clinical trial on 42 outpatients. The patients were treated with $20 \mathrm{mg}$ memantine daily (as was the case with Herrmann et al. ${ }^{28}$ ) for six months' duration. The standardized neuropsychiatric tests scores used to compare their baseline scores with endpoint scores after six months treatment reported much better improvements to memantine in patients with moderate-to-severe bvFTD, but found no beneficial effects of cognitive function and behavioral symptoms in patients with mild bvFTD. Obviously, the study report needs to be interpreted with care as the possibility of spontaneous symptoms resolution could upswing the data resulting in the statistically significant improvement in the moderate-to-severe bvFTD patients. Quite arguably as well, the lack of beneficial effects in patients with mild bvFTD may be related to compliance issues, particularly if the patients did not feel too unwell in themselves to justify the necessity for treatment compliance lasting six months period. More importantly, as claimed by the authors, the low power of the study also explains why extra caution must be used when applying the trial outcome to clinical practice.

In another study, a drug called mibampator (an AMPA receptor potentiator) was studied against placebo treatment in $132 \mathrm{AD}$ patients with agitation and aggression who were randomized to 12 weeks of double-blind treatment. ${ }^{30}$ The trial findings show no evidence of advantage of mibampator over placebo in treating agitation and aggression in AD patients

Further, in 2014, Porsteinsson et al. ${ }^{31}$ conducted a 9-week double-blind randomized trial on 186 probable AD patients with agitation across 8 academic centers in Canada and United States. They found that among the patients with probable Alzheimer disease-related agitation who were receiving psychosocial intervention, the addition of citalopram compared with placebo leads to significantly reduced agitation. However, citalopram dosage may have to be limited to $30 \mathrm{mg}$ per day due to worsening of cognition and worrying cardiac (QTc prolongation) adverse effects noted in patients. Porsteinsson et al. conclude that evidence available to assess the efficacy of citalopram at lower doses for treating agitation is insufficient. However, earlier in 2012, FDA ${ }^{58}$ updated their safety warnings that citalopram was associated the dose-dependent risk of QT prolongation which may cause Torsade's de Pointes, ventricular tachycardia and sudden death. They advised dose restriction with a maximum recommended dose of $20 \mathrm{mg}$ per day for patients who are older than 60 years; have liver impairments; or taking other drugs (CYP2C19 inhibitor) that can increase blood levels of citalopram. Drye et al. ${ }^{64}$ study data reinforce the FDA warning against the use of higher doses of citalopram in people over 60 years, and report that Citalopram, at $30 \mathrm{mg}$ per day, reduces agitation in patients with Alzheimer's disease but also causes concerning cognitive effects and QT prolongation.

Further study in 2016 by Ho et al. ${ }^{65}$ compared the relationship between (R) and (S)-citalopram enantiomer exposure and explored their therapeutic response on agitation behaviors in elderly $A D$ population. Their analysis showed evidence that (S)citalopram (escitalopram) is more efficacious than (R)-citalopram in the treatment of agitation in an elderly people with $A D$ patients. In addition, exposure to (R)-citalopram revealed association of more adverse effects compared with (S)-citalopram exposure which established significant therapeutic benefit and a better choice than racemic $(\mathrm{R})$ citalopram.

Meanwhile, in a published 10 -week placebo-controlled randomized clinical trial, Cummings et al. ${ }^{32}$ study 220 probable AD patients with agitation to assess the efficacy, safety and tolerability of dextromethorphan-quinidine sulfate. The combination of dextromethorphan-quinidine treatment compared with placebo demonstrated clinically relevant efficacy for agitation management and was also found to be generally well-tolerated. While some adverse events including falls, diarrhea and urinary tract infections were reported with the dextromethorphan-quinidine treatment, there was however, no associated cognitive impairment, sedation or clinically significant QTc prolongation.

It is good to note that several ongoing clinical trials are focusing on novel drugs for treating agitation in dementia population. 
Among these is the clinical trial by Intra-Cellular Therapies, who are conducting a multicenter, randomized, double-blind, placebo-controlled Phase 3 clinical trial on ITI-007-201 to evaluate the efficacy, safety and tolerability of ITI-007 in patients with a clinical diagnosis of probable $A D$ with clinically significant agitation. ${ }^{66}$ The study anticipates to enroll 360 subjects that will be randomly assigned to receive a one-month course of $9 \mathrm{mg}$ per day of ITI-007 against placebo. $^{66}$ Similarly, University of Sussex in United Kingdom is carrying out phase 3 clinical trial on mirtazapine or carbamazepine use for agitation in dementia (SYMBAD). ${ }^{67}$ The authors aim to assess whether Mirtazapine or Carbamazepine are more effective than placebo in treating agitation in people with dementia. The trial will assess the safety, clinical and cost effectiveness of each treatment over 12 weeks period and will follow the participants for up to one year. ${ }^{67}$

A phase 3, multicenter, randomized, double-blind, placebocontrolled study by Avanir Pharmaceuticals is also ongoing. ${ }^{68}$ The trial aims to assess the efficacy, safety and tolerability of AVP-786 (Deuterated [d6]-Dextromethorphan Hydrobromide [d6-DM]/Quinidine Sulfate [Q]) for the treatment of agitation in patients with dementia of the Alzheimer's Type. ${ }^{68}$ Otsuka Pharmaceutical is also conducting ongoing phase 3 trial to evaluate the efficacy, dose-response and safety of aripiprazole at 2, 3 and $6 \mathrm{mg}$ per day in comparison with placebo in patients with agitation associated with AD. ${ }^{69}$ Meanwhile, Acadia Pharmaceuticals phase 2 study is evaluating the efficacy of pimavanserin compared with placebo following 12 weeks of treatment for agitation and aggression in $\mathrm{AD} .^{70}$

When completed, these ongoing studies will hopefully achieve a balance of efficacy over safety for drugs used in treating dementia-related agitation and other neuropsychiatric symptoms.

\section{ANECDOTE OF CLINICAL EXPERIENCE}

Over the past three years, we have experience a surge in the number of dementia-related illnesses being admitted to our unit. Quite recently, we managed a clinical condition that apparently stimulates the urge for writing this review. The intention is to increase the awareness of fellow clinicians/physicians, and also, hoping the carers and family of dementia patients who read medical journals will be better informed.

An elderly frail woman, mostly bedbound, from a nursing home was admitted to our unit. She had background history of frontotemporal dementia and previous stroke. Her family mentioned that she often became agitated whenever she had exacerbations of arthritic pains or infections (particularly urinary infection). However, due to rising frequencies of agitation and aggression symptoms in the nursing home, she was reviewed by the community Psychiatry team and was started on quetiapine treatment. On subsequent review by the latter, risperidone was added to her medications due to lack of improvement of her symptoms. The addition of risperidone made her to be overly drowsy on most time of the day, and her oral intake became very poor according to her family.

Unfortunately, in just over six months on quetiapine and risperidone combination, she suffered another major ischemic stroke, and was started on end of life care in the same nursing home. At that time, all her active medications were stopped including the antipsychotics (risperidone and quetiapine). Surprisingly, she made a spontaneous recovery after few weeks, and her oral intake began to improve at the time. However, about two months following her recovery, she was restarted on her regular medications including the antipsychotics. Again, she started becoming drowsy again at most times of the day with worsening oral intake due to drowsiness. Little wonder, she presented to our unit with acute kidney injury from severe dehydration and also had aspiration pneumonia. During the week-long period of admission on our unit, her antipsychotics medications were stopped and she was rehydrated and treated for likely aspiration pneumonia. Her oral intake became significantly improved and she was neither agitated nor aggressive at any time during the period of her admission into our unit. On discharge, I discussed with the patient's next of kin the need to stop both the risperidone and quetiapine, as these medications were no longer indicated in the patient. I also put a note to the psychiatry team to inform them of the above plan and requested a follow-up by them.

From the above case, it is debatable whether or not the antipsychotic medications were the cause of her second stroke or perhaps, contributed to its occurrence. Also, the dehydration and likely aspiration pneumonia might arguably be connected to the excessive sedative effects of her antipsychotics medications. It is also unclear how much training the nursing home staffs had in caring for dementia patients generally, and how much of non-pharmacological approach was recommended by the psychiatry team before the start of the antipsychotic medications whose efficacy was questionable and with proven adverse effects?

Not surprisingly, van der Ploeg et al. ${ }^{71}$ in their research on 17 aged care facilities in Southeast Melbourne, Australia, report that non-pharmacological interventions are rarely implemented in aged care facilities despite their proven beneficial effects for agitated patients. They cited staff members' lack of time as the main reason for the poor implementation of non-pharmacological interventions. Unfortunately, the above reason might just be applicable to hospitals as well, explaining why many clinicians have low threshold for commencing drug treatment at the slightest presentation of agitation by dementia patients, lacking the patience to embrace non-pharmacological approach as the initial first line treatment interventions. Some clinicians even commence dementia patients with previous history of agitation or other neuropsychiatric symptoms on longstanding antipsychotics and/or anxiolytics/hypnotics drug as maintenance treatment, ignoring several warnings by FDA and recommendations from many associations of geriatrics and psychiatry societies advising on careful use of drug interventions.

\section{CONCLUSION}

As dementia is increasingly becoming more prevalent in this day and age, unfortunately, the treatment of dementia-related agitation and other behavioral symptoms have been a challenge. All the potential pharmacological treatment interventions present risks as well as concerns on their efficacy. Some pharmacotherapy of proven effectiveness, such as dextromethorphan-quinidine are not without safety concerns. Likewise, the non-pharmacological management which is the accepted first-line treatment approach may also not be effective in managing severe agitation and other behavioral symptoms in every dementia patient.

Future studies will be needed to focus on exploring the efficacy of non-pharmacological interventions and palliative care approach in advanced dementia, as well as determining the safety, tolerability and efficacy of the drugs treatment in managing the neuropsychiatric symptoms of dementia.

This review provides opportunity for increase knowledge and awareness of the non-pharmacological interventions among physicians, especially the clinicians with no specialty training in geriatrics medicine but, see dementia patients with agitation and other behavioral symptoms from time to time. Likewise, it hopefully will benefit all the readers of medical sciences to update their existing knowledge on matters relating to the management of dementia-related agitation. 


\section{CONFLICT OF INTEREST}

The author declare no conflict of interest.

\section{ACKNOWLEDGMENTS}

I appreciate the support and guidance from Drs Robert and Elizabeth Eilers of Drexel University College of Medicine (Graduate Research Program), Philadelphia, USA, during the writing of the review. Also, special thank goes to Mr John Hudson of Bell library at Royal Wolverhampton NHS Trust, Wolverhampton, UK, for his support with the references. Finally, to my darling wife, Dr Ruth ljaopo and my two kids, I appreciate your immeasurable support and words of encouragement at all times.

\section{REFERENCES}

1 Prince M, Wimo A, Guerchet M, Ali G, Wu Y, Prina M. World Alzheimer Report 2015. The global impact of dementia. An analysis of prevalence, incidence, cost \& trends. London: Alzheimer's Disease International (ADI); August 2015.

2 Association As. Alzheimer's disease facts and figures. Alzheimer's Dement: J Alzheimer's Assoc 2014; 10: e47-e92.

3 Hurd MD, Martorell P, Delavande A, Mullen KJ, Langa KM. Monetary costs of dementia in the United States. N Engl J Med 2013; 368: 1326-1334.

4 Association As Alzheimer's disease facts and figures. Alzheimer's Dement 2010; 6: 158-194.

5 Ballard C, Lana MM, Theodoulou M, Douglas S, McShane R, Jacoby R et al. A randomised, blinded, placebo-controlled trial in dementia patients continuing or stopping neuroleptics (the DART-AD trial). PLoS Med 2008; 5: e76.

6 Casey DA. Pharmacotherapy of neuropsychiatric symptoms of dementia. $P \& T$ : Peer-rev J Formul Manag 2015; 40: 284-287.

7 Lyketsos CG, Carrillo MC, Ryan JM, Khachaturian AS, Trzepacz P, Amatniek J et al. Neuropsychiatric symptoms in Alzheimer's disease. Alzheimer's Dement 2011; 7: 532-539.

8 Oliveira AMD, Radanovic M, Mello PCHD, Celestino DL, Forlenza OV, Buchain PC et al. Nonpharmacological interventions to reduce behavioral and psychological symptoms of dementia: a systematic review. BioMed Res Int 2015; 2015: 218980.

9 Acharya D, Harper DG, Achtyes ED, Seiner SJ, Mahdasian JA, Nykamp LJ et al. Safety and utility of acute electroconvulsive therapy for agitation and aggression in dementia. Int J Geriatr Psychiatry 2015; 30: 265-273.

10 Jakobson E, Avari J, Kalayam B. Non-pharmacologic interventions for treatment of agitation in dementia in nursing home residents. Am J Geriatr Psychiatry 2015; 23 (3 Suppl. 1): S139-S140.

11 Yang MH, Lin LC, Wu SC, Chiu JH, Wang PN, Lin JG. Comparison of the efficacy of aroma-acupressure and aromatherapy for the treatment of dementia-associated agitation. BMC Complement Altern Med 2015; 15: 93.

12 Hendriks SA, Smalbrugge M, Hertogh CMPM, van der Steen JT, Galindo-Garre F. From admission to death: prevalence and course of pain, agitation, and shortness of breath, and treatment of these symptoms in nursing home residents with dementia. J Am Med Dir Assoc 2015; 16: 475-481.

13 Gitlin LN, Kales HC, Lyketsos CG. Nonpharmacologic management of behavioral symptoms in dementia. J Am Med Assoc 2012; 308: 2020-2029.

14 Ridder HM, Stige B, Qvale LG, Gold C. Individual music therapy for agitation in dementia: an exploratory randomized controlled trial. Aging Mental Health 2013; 17: 667-678.

15 Hawranik P, Johnston P, Deatrich J. Therapeutic touch and agitation in individuals with Alzheimer's disease. West J Nurs Res 2008; 30: 417-434.

16 Woods DL, Craven RF, Whitney J. The effect of therapeutic touch on behavioral symptoms of persons with dementia. Altern Ther Health Med 2005; 11: 66.

17 O'Connor DW, Eppingstall B, Taffe J, van der Ploeg ES. A randomized, controlled cross-over trial of dermally-applied lavender (Lavandula angustifolia) oil as a treatment of agitated behaviour in dementia. BMC Complement Altern Med 2013; 13: 315 .

18 Livingston G, Kelly L, Lewis-Holmes E, Baio G, Morris S, Patel N et al. Nonpharmacological interventions for agitation in dementia: systematic review of randomised controlled trials. Br J Psychiatry 2014; 205: 436-442.

19 Abraha I, Rimland JM, Trotta FM, Dell'Aquila G, Cherubini A, Cruz-Jentoft A et al. Systematic review of systematic reviews of non-pharmacological interventions to treat behavioural disturbances in older patients with dementia. The SENATOROnTop series. BMJ Open 2017; 7: e012759.

20 Abraha I, Rimland JM, Lozano-Montoya I, Dell'Aquila G, Velez-Diaz-Pallares M, Trotta FM et al. Simulated presence therapy for dementia. Cochrane Datab Syst Rev 2017; 4: Cd011882.

21 Vink AC, Slaets JPJ, Zuidersma M, De Jonge P, Boersma F, Zuidema SU. The effect of music therapy compared with general recreational activities in reducing agitation in people with dementia: a randomised controlled trial. Int J Geriatr Psychiatry 2013; 28: 1031-1038.
22 Jutkowitz E, Brasure M, Fuchs E, Shippee T, Kane RA, Butler M et al. Care-delivery interventions to manage agitation and aggression in dementia nursing home and assisted living residents: a systematic review and meta-analysis. J Am Geriatr Soc 2016; 64: 477-488.

23 Rappaport SA, Marcus RN, Manos G, Oren DA, McQuade RD. A randomized, double-blind, placebo-controlled tolerability study of intramuscular aripiprazole in acutely agitated patients with alzheimer's, vascular, or mixed dementia. J Am Med Dir Assoc 2009; 10: 21-27.

24 Cakir S, Kulaksizoglu IB. The efficacy of mirtazapine in agitated patients with Alzheimer's disease: a 12-week open-label pilot study. Neuropsychiatr Dis Treat 2008; 4: 963-966.

25 Wang LY, Shofer JB, Rohde K, Hart KL, Hoff DJ, McFall YH et al. Prazosin for the treatment of behavioral symptoms in patients with Alzheimer disease with agitation and aggression. Am J Geriatr Psychiatry 2009; 17: 744-751.

26 Lockhart IA, Orme ME, Mitchell SA. The efficacy of licensed-indication use of donepezil and memantine monotherapies for treating behavioural and psychological symptoms of dementia in patients with Alzheimer's disease: systematic review and meta-analysis. Dement Geriatr Cogn Dis Extra 2011; 1: 212-227.

27 Fox C, Crugel M, Maidment I, Auestad BH, Coulton S, Treloar A et al. Efficacy of memantine for agitation in Alzheimer's dementia: a randomised double-blind placebo controlled trial. PLOS ONE 2012; 7: e35185.

28 Herrmann N, Gauthier S, Boneva N, Lemming OM. A randomized, double-blind, placebo-controlled trial of memantine in a behaviorally enriched sample of patients with moderate-to-severe Alzheimer's disease. Int Psychogeriatr 2013; 25: 919-927.

29 Li P, Quan W, Zhou Y-Y, Wang Y, Zhang H-H, Liu S. Efficacy of memantine on neuropsychiatric symptoms associated with the severity of behavioral variant frontotemporal dementia: a six-month, open-label, self-controlled clinical trial. Exp Ther Med 2016; 12: 492-498.

30 Trzepacz PT, Cummings J, Konechnik T, Forrester TD, Chang C, Dennehy EB et al. Mibampator (LY451395) randomized clinical trial for agitation/aggression in Alzheimer's disease. Int Psychogeriatr 2013; 25: 707-719.

31 Porsteinsson AP, Drye LT, Pollock BG, Devanand DP, Frangakis C, Ismail Z et al. Effect of citalopram on agitation in Alzheimer disease: the CitAD randomized clinical trial. J Am Med Assoc 2014; 311: 682-691.

32 Cummings JL, Lyketsos CG, Peskind ER, Porsteinsson AP, Mintzer JE, Scharre DW et al. Effect of Dextromethorphan-Quinidine on agitation in patients with alzheimer disease dementia: a randomized clinical trial. J Am Med Assoc 2015; 314: 1242-1254.

33 NICE. Management of aggression, agitation and behavioural disturbances in dementia: valproate preparations. Evidence summary: unlicensed or off-label medicine. National Institute for Health and Clinical Excellence (ESUOM41), 2015.

34 Ayalon L, Gum AM, Feliciano L, Arean PA. Effectiveness of nonpharmacological interventions for the management of neuropsychiatric symptoms in patients with dementia: a systematic review. Arch Intern Med 2006; 166: 2182-2188.

35 Kales HC, Gitlin LN, Lyketsos CG. Management of neuropsychiatric symptoms of dementia in clinical settings: recommendations from a multidisciplinary expert panel. J Am Geriatr Soc 2014; 62: 762-769.

36 Passmore MJ, Gardner DM, Polak Y, Rabheru K. Alternatives to atypical antipsychotics for the management of dementia-related agitation. Drugs Aging 2008; 25: 381-398.

37 Lichtwarck B, Selbaek G, Kirkevold $\varnothing$, Rokstad AMM, Benth J, Myhre J et al. TIME Targeted interdisciplinary model for evaluation and treatment of neuropsychiatric symptoms: protocol for an effectiveness-implementation cluster randomized hybrid trial. BMC Psychiatry 2016; 16: 233.

38 Cohen-Mansfield J, Jensen B, Resnick B, Norris M. Knowledge of and attitudes toward nonpharmacological interventions for treatment of behavior symptoms associated with dementia: a comparison of physicians, psychologists, and nurse practitioners. Gerontologist 2012; 52: 34-45.

39 Rapp MA, Mell T, Majic T, Treusch Y, Nordheim J, Niemann-Mirmehdi M et al. Agitation in nursing home residents with dementia (VIDEANT trial): effects of a cluster-randomized, controlled, guideline implementation trial. J Am Med Dir Assoc 2013; 14: 690-695.

40 Blythe SL, Chang E, Johnson A, Griffiths R. The efficacy of nurse implemented nonpharmacological strategies for the symptom management of agitation in persons with advanced dementia living in residential aged care facilities: a systematic review. JBI Lib Syst Rev 2009; 7: 975-1003.

41 Cooke ML, Moyle W, Shum DH, Harrison SD, Murfield JE. A randomized controlled trial exploring the effect of music on agitated behaviours and anxiety in older people with dementia. Aging Mental Health 2010; 14: 905-916.

42 Lin PWK, Ng BFL, Chan WC, Lam LCW. Efficacy of aromatherapy (Lavandula angustifolia) as an intervention for agitated behaviours in Chinese older persons with dementia: a cross-over randomized trial. Int J Geriatr Psychiatry 2007; 22: $405-410$. 
43 Van Der Ploeg ES, Eppingstall B, Runci SJ, O'Connor DW, Camp CJ, Taffe J. A randomized crossover trial to study the effect of personalized, one-to-one interaction using Montessori-based activities on agitation, affect, and engagement in nursing home residents with dementia. Int Psychogeriatr 2013; 25: 565-575.

44 Van Vracem M, Spruytte N, Declercq A, Van Audenhove C. Agitation in dementia and the role of spatial and sensory interventions: experiences of professional and family caregivers. Scand J Caring Sci 2016; 30: 281-289.

45 Lin LC, Yang MH, Kao CC, Wu SC, Tang SH, Lin JG. Using acupressure and Montessori-based activities to decrease agitation for residents with dementia: a cross-over trial. J Am Geriatr Soc 2009; 57: 1022-1029.

46 Raglio A, Bellelli G, Traficante D, Gianotti M, Ubezio MC, Villani D et al. Efficacy of music therapy in the treatment of behavioral and psychiatric symptoms of dementia. Alzheimer Dis Assoc Disord 2008; 22: 158-162.

47 Sung HC, Chang AM. Use of preferred music to decrease agitated behaviours in older people with dementia: a review of the literature. J Clin Nurs 2005; 14: $1133-1140$.

48 Gitlin LN, Winter L, Burke J, Chernett N, Dennis MP, Hauck WW. Tailored activities to manage neuropsychiatric behaviors in persons with dementia and reduce caregiver burden: a randomized pilot study. Am J Geriatr Psychiatry 2008; 16: 229-239.

49 Koulivand PH, Khaleghi Ghadiri M, Gorji A. Lavender and the nervous system. Evid Based Complement Alternat Med 2013; 2013: 681304.

50 Jain NL, Ehwarieme R, Kusz HG. Dementia related behavioral problems: when less can do more in managing a difficult patient. J Am Geriatr Soc 2015; 63(Supplement S1): S240.

51 Ray KD, Mittelman MS. Music therapy: a nonpharmacological approach to the care of agitation and depressive symptoms for nursing home residents with dementia. Dementia 2015; 16: 689-710.

52 Janzen S, Zecevic AA, Kloseck M, Orange JB. Managing agitation using nonpharmacological interventions for seniors with dementia. Am J Alzheimers Dis Other Dement 2013; 28: 524-532.

53 Cohen-Mansfield J, Juravel-Jaffe A, Cohen A, Rasooly I, Golander H. Physicians' practice and familiarity with treatment for agitation associated with dementia in Israeli nursing homes. Int Psychogeriatr 2013; 25: 236-244.

54 Kverno KS, Black BS, Nolan MT, Rabins PV. Research on treating neuropsychiatric symptoms of advanced dementia with non-pharmacological strategies, 1998-2008: a systematic literature review. Int Psychogeriatr 2009; 21: 825-843.

55 Eapcnet.eu. Recommendations on palliative care and treatment of older people with Alzheimer's disease and other progressive dementias. European Association for Palliative Care; 2013.

56 Van Der Steen JT, Hertogh CM, De Boer ME, Francke AL, Junger S, Radbruch L et al. White paper defining optimal palliative care in older people with dementia: a Delphi study and recommendations from the European Association for Palliative Care. Palliat Med 2014; 28: 197-209.

57 Hendriks SA, Smalbrugge M, Hertogh CMPM, Van Der Steen JT. Dying with dementia: symptoms, treatment, and quality of life in the last week of life. $J$ Pain Symptom Manage 2014; 47: 710-720.

58 Food \& Drug Administration. FDA Drug Safety Communication: Revised recommendations for Celexa (citalopram hydrobromide) related to a potential risk of abnormal heart rhythms with high doses. US Food and Drug Administration: Rockville, MD, 2012.

59 Seitz DP, Gill SS, Herrmann N, Brisbin S, Rapoport MJ, Rines J et al. Pharmacological treatments for neuropsychiatric symptoms of dementia in long-term care: a systematic review. Int Psychogeriatr 2013; 25: 185-203.

60 Schneider LS, Dagerman KS, Insel P. Risk of death with atypical antipsychotic drug treatment for dementia: meta-analysis of randomized placebo-controlled trials. J Am Med Assoc 2005; 294: 1934-1943.
61 Gill SS, Bronskill SE, Normand SL, Anderson GM, Sykora K, Lam K et al. Antipsychotic drug use and mortality in older adults with dementia. Ann Intern Med 2007; 146: 775-786.

62 Herrmann N, Lanctot KL. Do atypical antipsychotics cause stroke? CNS Drugs 2005; 19: 91-103.

63 Jalbert JJ, Eaton CB, Miller SC, Lapane KL. Antipsychotic use and the risk of hip fracture among older adults afflicted with dementia. J Am Med Dir Assoc 2010; 11: 120-127.

64 Drye LT, Spragg D, Devanand DP, Frangakis C, Marano C, Meinert CL et al. Changes in QTc interval in the citalopram for agitation in Alzheimer's disease (CitAD) randomized trial. PLOS ONE 2014; 9: e98426.

65 Ho T, Schantz O, Bies R, Pollock BG, Mulsant BH, Devanand DP et al. R- and S-citalopram concentrations have differential effects on neuropsychiatric scores in elders with dementia and agitation. Br J Clin Pharmacol 2016; 82: 784-792.

66 Intra-Cellular Therapies, Inc. ITI-007 for the Treatment of Agitation in Patients With Dementia, Including Alzheimer's Disease. (ClinicalTrials.gov National Library of Medicine (US), [Internet] Bethesda (MD), 2008. [Cited April 10, 2017]. Available from https://clinicaltrials.gov/ct2/show/NCT02817906.

67 University of Sussex. A Pragmatic, Multi Centre, Double-blind, Placebo Controlled Randomised Trial to Assess the Safety, Clinical and Cost Effectiveness of Mirtazapine or Carbamazepine in Patients With Alzheimer's Disease (AD) and Agitated Behaviours (ClinicalTrials.gov National Library of Medicine (US), [Internet] Bethesda (MD) (2008) [Cited April 2, 2017]. Available from https://clinicaltrials.gov/ct2/show/ NCT03031184.

68 Avanir Pharmaceuticals. A Phase 3, Multicenter, Randomized, Double-blind, Placebocontrolled Study to Assess the Efficacy, Safety, and Tolerability of AVP-786 (Deuterated [d6]-Dextromethorphan Hydrobromide [d6-DM]/Quinidine Sulfate [Q]) for the Treatment of Agitation in Patients With Dementia of the Alzheimer's Type (ClinicalTrials.gov National Library of Medicine (US), [Internet]. Bethesda (MD) (2017), [Cited April 10, 2017]. Available from https://clinicaltrials.gov/ct2/show/ NCT02442765.

69 Otsuka Pharmaceutical Co., Ltd. A Multicenter, Randomized, Double-blind, Placebocontrolled Trial to Evaluate the Efficacy, Safety, and Tolerability of Aripiprazole in the Treatment of Patients With Agitation Associated With Dementia of the Alzheimer's Type (ClinicalTrials.gov National Library of Medicine (US), [Internet] Bethesda (MD) (2017). [Cited April 10, 2017]. Available from https://clinicaltrials.gov/ct2/show/ NCT02168920.

70 Acadia Pharmaceuticals Inc. A Double-Blind, Placebo-Controlled Study to Examine the Safety and Efficacy of Pimavanserin for the Treatment of Agitation and Aggression in Alzheimer's Disease (ClinicalTrials.gov National Library of Medicine (US), [Internet] Bethesda (MD), (2016). [Cited April 5, 2017]. Available from https:// clinicaltrials.gov/ct2/show/NCT02992132.

71 van der Ploeg ES, Mbakile T, Genovesi S, O'Connor DW. The potential of volunteers to implement non-pharmacological interventions to reduce agitation associated with dementia in nursing home residents. Int Psychogeriatr 2012; 24: 1790-1797.

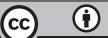

This work is licensed under a Creative Commons Attribution 4.0 International License. The images or other third party material in this article are included in the article's Creative Commons license, unless indicated otherwise in the credit line; if the material is not included under the Creative Commons license, users will need to obtain permission from the license holder to reproduce the material. To view a copy of this license, visit http://creativecommons.org/licenses/ by/4.0/

(c) The Author(s) 2017 